
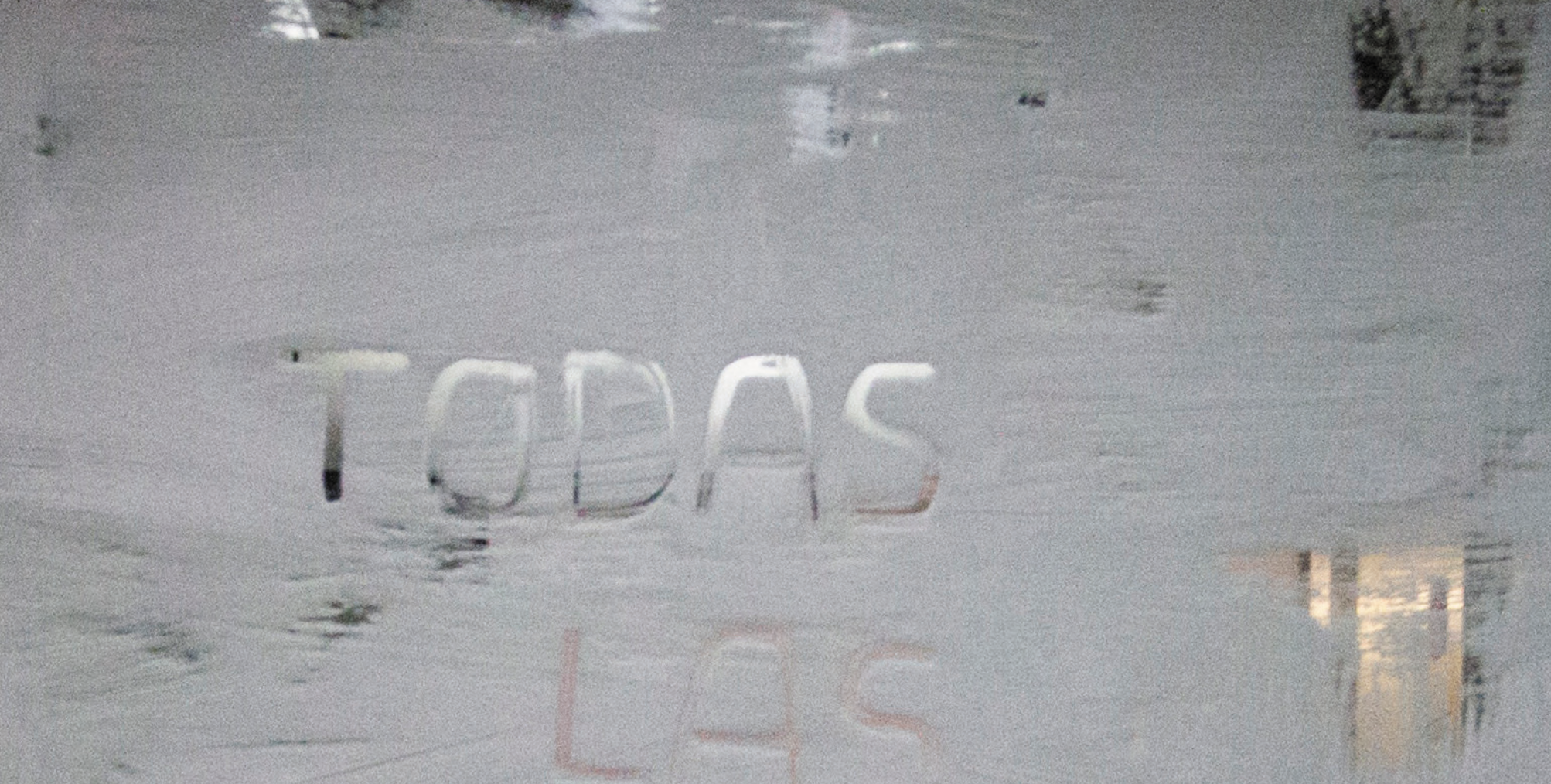

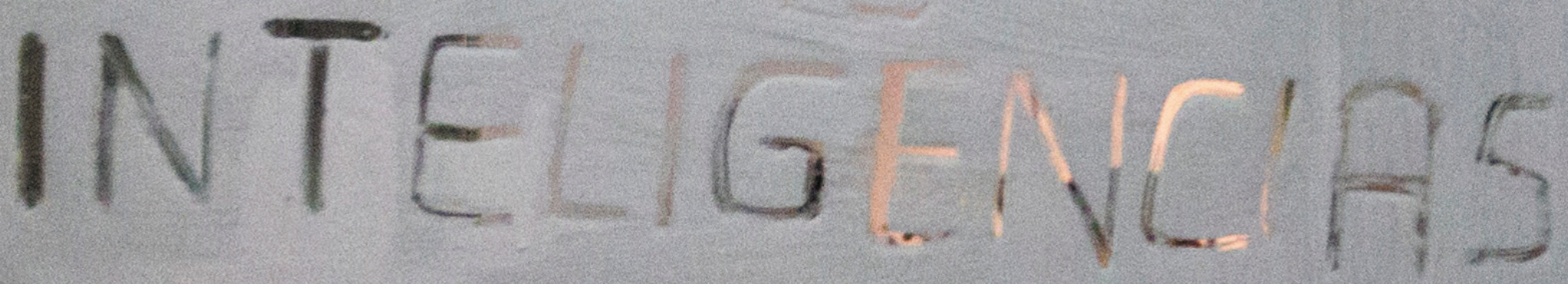
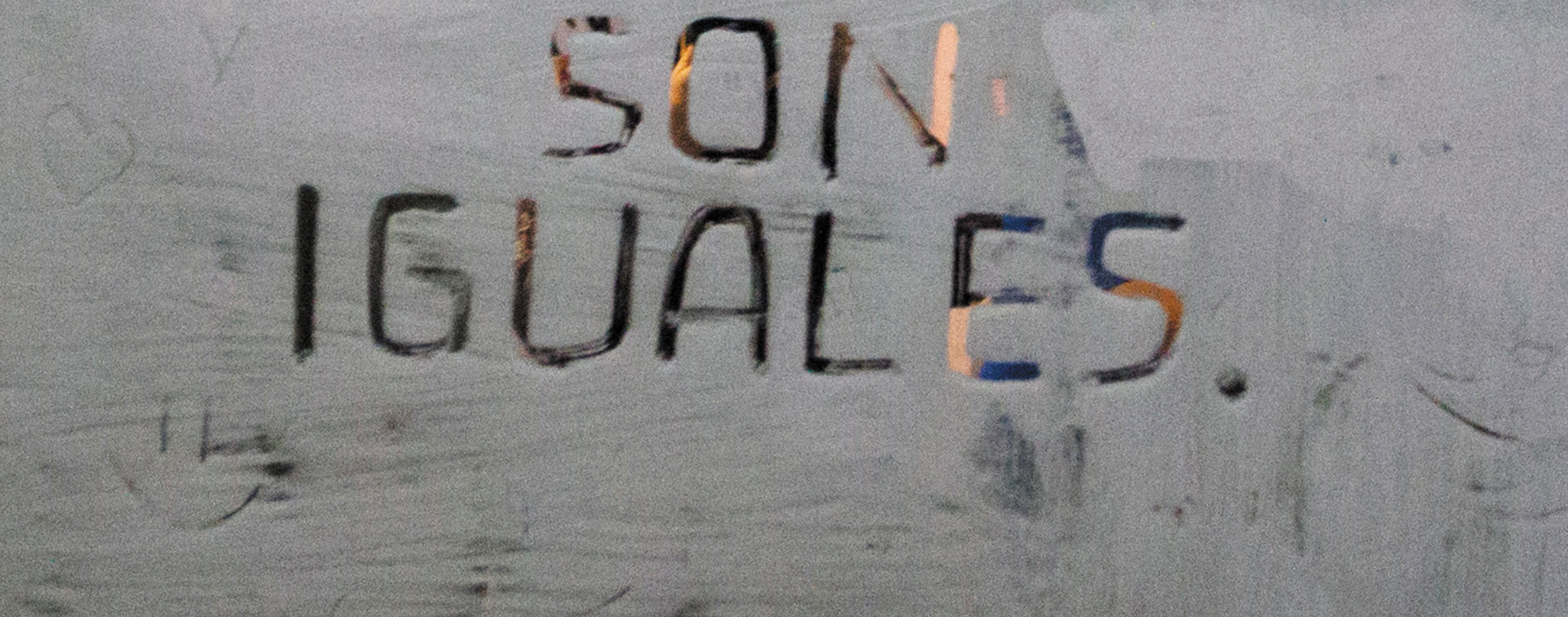

(⿸丆口

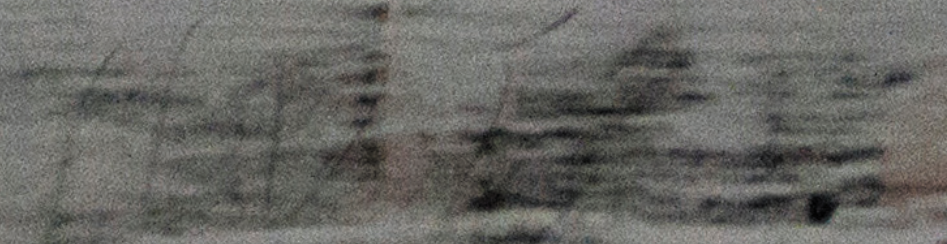

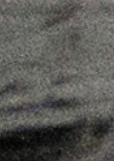
(2)

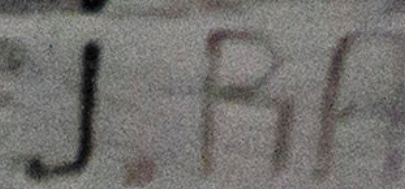

NE

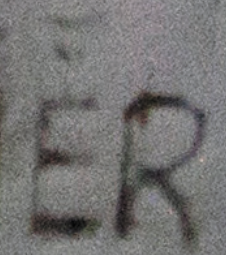

1. 


\section{Trabajo, cotidianeidad, ocupación y cooperativismo en Casa Tomada}

\section{Artículo de reflexión}

\section{Victoria Rodríguez do Campo}

Universidad de Buenos Aires

victoriardc@gmail.com

Cómo citar éste artículo: Rodríguez V. [2017]. Trabajo, cotidianidad, ocupación y cooperativismo en CASA TOMADA, Calle14 revista de investigación en el campo del arte, 12[२], 292-301

DOl: https://doi.org/10.14483/21450706.12360

\section{Resumen}

El proyecto artístico interdisciplinario Casa Tomada opera como disparador para el abordaje de problemáticas propias de la coyuntura social y artística contemporánea. La ficción creada por la Casa Nacional del Bicentenario, espacio cultural de la Ciudad de Buenos Aires, abre el camino a considerar formas de creación alternativas en las que se pone en jaque el estatuto del trabajo del artista y se vislumbran renovados intersticios a través de la acción de los múltiples actuantes que circundan el proyecto. Con la acción política ilícita como puntapié inicial -la ocupación forzada de un espacio público - Casa Tomada apuesta por la mostración de una multiplicidad de conflictos, tensiones, preguntas, así como posibles respuestas, siempre contingentes, aplicables tanto al ámbito social como al artístico.

\section{Palabras claves}

Arte contemporáneo, ocupación, política, trabajo colectivo, interdisciplinariedad. 


\section{Work, Dialogue, Occupation and Cooperativism at Casa Tomada}

Abstract

The interdisciplinary art project Casa Tomada operates as a trigger for addressing issues of the social and artistic contemporary juncture. The fiction created by the National House of the Bicentennial, cultural space of the City of Buenos Aires, opens the way to consider alternative forms of creation in which the status of the artist's work is put in check and renewed interstices are glimpsed through the action of the multiple actors that surround the project. With illegal political action as a starting point the forced occupation of a public space, Casa Tomada is committed to showing a multiplicity of conflicts, tensions, questions as well as possible answers, which are always contingent and applicable both to the social and the artistic spheres.

\section{Keywords}

Contemporary art, occupation, politics, collective work, interdisciplinarity.

\section{Travail, dialogue, occupation et coopératisme à Casa Tomada}

\section{Résumé}

Le projet d'art interdisciplinaire Casa Tomada fonctionne comme un déclencheur pour résoudre les problèmes dérivés de la situation social et artistique contemporaine. La fiction créée par la Maison Nationale du Bicentenaire, espace culturel de la Ville de Buenos Aires, ouvre la voie à la recherche de formes alternatives de création dans lesquelles le statut de l'œuvre de l'artiste est mis en échec et des interstices renouvelés sont visibles par action des acteurs multiples qui entourent le projet. Avec l'action politique illégale comme point de départ -l'occupation forcée d'un espace public- Casa Tomada est engagée à montrer une multiplicité des conflits, des tensions, des questions et de possibles réponses, toujours contingentes, applicables à la fois au champ social et à l'artistique.

\section{Mots clés}

Art contemporain, profession, politique, travail collectif, interdisciplinarité.

\section{Trabalho, cotidianeidade, ocupação e cooperativismo em casa tomada}

\section{Resumo}

O projeto artístico interdisciplinar de Casa Tomada opera como disparador para a abordagem de problemáticas próprias da conjuntura social e artística contemporânea. A ficção criada pela Casa Nacional do Bicentenário, espaço cultural da Cidade de Buenos Aires, abre o caminho a considerar formas de criação alternativas nas que se põe cheque mate o estatuto do trabalho do artista e se vislumbram renovados interstícios através da ação dos múltiplos atuantes que circundam o projeto. Com a ação política ilícita como pontapé inicial -a ocupação forçada de um espaço público - Casa Tomada aposta pela mostra de uma multiplicidade de conflitos, tensões, perguntas, assim como possiveis respostas, sempre contingentes, aplicáveis tanto ao âmbito social como ao artístico.

\section{Palavras-chave}


Arte contemporânea, ocupação, política, trabalho coletivo, interdisciplinariedade.

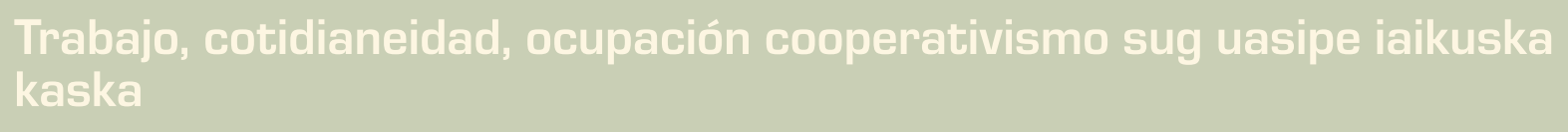

\section{Maillallachiska:}

Chi proyecto artístico interdisciplinario sug uasipe iaikuska kaska sug togiachidorsina abordajepe problematikakuna chi coyuntura social y artística contemporánea. Chi ficción uiñachiska uasi nacional Bicenterariopa, luar cultural Buenos Aires ciudadmanda,mpaskan ñambe consideraspa sug uiñachiskakuna alternativa churra jaque estatuto trabajope artistapa y vislumbrarse renovado intersticiokuna multiple actuantekuna que circundadn el proyecto. Chi acción política ilicitak sug artagsina inicial- chi ocupación fuerzaskak sug luar publicocasa tomada apuesta, kauachiska sug multiplicidad de conflictos, tensiones, tapuchiikuna, chasallata sug aineikuna, siempre contingente aplicables tanto al ámbito social como artístico.

\section{Rimangapa Ministidukuna:}

Arte contemporáneo ocupación, política, trabajo colectivo, interdisciplinariedad.

\section{Introducción}

Casa Tomada se erige como un proyecto multidisciplinario que incluye las creaciones de más de sesenta artistas ${ }^{1}$, quienes vivieron y trabajaron, entre agosto y diciembre de 2016, en la Casa Nacional del Bicentenario ${ }^{2}$, organismo público y de gestión estatal, ubicado en Riobamba 985 , zona céntrica de la Ciudad de Buenos Aires. Las formas

1 Entre ellos Alan Courtis, Alejo Moguillansky, Alejo Wilkinson, Archivo Puerto Piojo, AVH, Carolina Andreeti, Bernardo Clausi, Biblioteca Popular Ambulante, Bustos + Galay, Casa de la Paz, Ciro Múseres, Claudia Cortínez, Club del Super 8, David López Mastrangelo, Elena Dahn, El Rancho Urbano, Ernesto Romeo, Fabro Tranchida, Federico Luna, Fernando Brizuela, Gabriel Baggio, Gabriel Chwojnik, Gabriel Saie, Gabriela Pulopulo, Informe Capital: Karina Granieri y Alicia Herrero, Javier Plano, Jésica Josiowicz, Jimena Croceri, Jorge Crowe, Juan Gugger, Julián D’Angiolillo, Julia Cossani, Leila Córdoba, Leonello Zambón, Lisandro Alonso, Lisandro Rodríguez, Lucas Di Pascuale, Luciana Acuña, Luis Terán, Luján Funes, M7Red, Martín Seijo, Matías Ibarra, Manuel Osorio, Matthieu Perpoint, Mil Focos, Nair Noronha, Pablo Méndez, Romina Orazi, Roger Colom, Rodo Túnica, Sebastián Friedman, SonidoCínico, Saya Sathya, Sofía Durrieu, Sofía Mazza, Sonidos Mutantes, TAPP, Telescuela Técnica, TRRUENO, Verónica Gómez, Yaeltex, Yaya Firpo. 2 En adelante CNB. de abordar el proyecto son múltiples. Cóctel de exhibición artística, performance, taller permanente, espacio de conciertos, cursos, charlas y proyecciones, Casa Tomada se escapa a la simple nominación. El proyecto ficcionaliza la ocupación forzada de la CNB por parte de los artistas, y aboga por un rompimiento de las estructuras tradicionales de las instituciones artísticas, invitando a los espectadores y a los trabajadores del espacio a arremangarse y crear a la par de los artistas. La labor y el rol social de estos artistascreadores-trabajadores-ocupantes también estará bajo la lupa. En este trabajo nos proponemos delinear algunos ejes sobre los cuales se construye el proyecto, buscando definir en qué intersticios radica su excepcionalidad, así como cuáles son sus aportes a la escena contemporánea local.

\section{Aproximaciones}

Es posible anidar esta experiencia con numerosas referencias del pasado. En principio, resulta inevitable vincular el fulgor antiinstitucional de Casa Tomada con los planteos de las vanguardias históricas de principios del siglo XX. Sin embargo, cabe preguntarse si dicho lazo resulta válido al referirse al caso argentino. 
Andrea Giunta se pregunta acerca del alcance de la vanguardia latinoamericana definida centralmente por su antiinstitucionalismo cuando las instituciones o bien no existían o se originaban al mismo tiempo que los gestos y las estrategias de vanguardia [Giunta, 2009: 143]. Un siglo después de dichas iniciativas, pasando por un proceso de paulatino y creciente establecimiento de instituciones artísticas en el territorio local, ¿Cómo puede concebirse el gesto antiinstitucional? Vale destacar que Casa Tomada se enfrenta a la Institución Arte sin salirse de los límites del espacio físico de dicho establecimiento y la acción de tomar el lugar no es más que una ficcionalización. Entonces, ¿invalidan estas circunstancias el valor de este proyecto? ¿Cuál es el sentido de crear una muestra que deja en jaque los preceptos básicos de la Institución Arte en la actualidad? ¿Por qué se elige ficcionalizar esa experiencia? Buscaremos acercarnos a estas cuestiones en las próximas páginas.

En relación a la idea del artista creando en la sala de exhibición, en el mismo espacio en el que el espectador verá la obra, realizando su labor en condiciones de sitespecific, podemos rastrear gran cantidad de iniciativas, partiendo del puntapié mentado por el movimiento minimalista. En el ámbito local, durante los último años, la Fundación Osde creó un espacio que opera en estas condiciones. El proyecto Intervención en salitas consiste en la exhibición del proceso de trabajo de un artista a lo largo de varios días, hasta que este queda finalizado para la contemplación convencional de la obra. Algo similar sucede con el Espacio Contemporáneo inaugurado en el último piso de Fundación Proa.

Como dijimos, Casa Tomada también imita, ficcionaliza una acción política. Este gesto de utilizar como vehículo de creación la simulación de herramientas de lucha y protesta de la sociedad contemporánea posee diversos antecedentes en la historia artística local en ejemplos como El que calla Otorga, Grupo de arte Callejero [GAC], Etcétera, o G.R.A.S.A., como caso más reciente. Estos grupos formulan acciones políticas que evidencien "la indiferenciación de los conceptos de militancia y práctica artística, la apropiación del espacio público y el carácter anónimo de sus propuestas" [AAV, 2010: 142].

Fuera de la ficción artística, podemos mencionar la acción de "tomar" un espacio como acto de apropiación política de gran profusión en el ámbito social y artístico de los últimos años. La multiplicidad de casos de fábricas tomadas y recuperadas por sus trabajadores implica una desjerarquización de las estructuras de poder, de los roles y los mandatos de las empresas. Las sucesivas crisis económicas y sociales, la lógica del mercado que prioriza los capitales concentrados antes que el bienestar del trabajador desembocó en un paulatino accionar por parte de los individuos afectados que consiste principalmente en la concepción de diversas formas de trabajo autogestionadas. La proliferación de organizaciones cooperativas en el territorio local es notable en las últimas décadas [Carman y Yacovino, 2007]. Por otro lado, la toma de un espacio se volvió una herramienta poderosa y habitual en la protesta educativa, sobre todo en el ámbito universitario e intelectual ${ }^{3}$. En los círculos propiamente artísticos a nivel internacional se destaca el caso del Teatro Valle Ocupatto de Roma ${ }^{4}$, tomado por artistas desde 2011, y el Cine Palazzo ${ }^{5}$, ubicado en la misma ciudad. Por otro lado, existen casos de espacios abandonados y posteriormente gestionados por artistas a través del apoyo o intervención del Estado, como La Tabacalera ${ }^{6}$ de Madrid o el proyecto ZKU7 de Berlín. En el espacio local, puede mencionarse la IMPA, que cumple el doble rol de fábrica recuperada por sus trabajadores para la producción de metales y plásticos, y, a su vez, de institución artístico cultural con múltiples ofertas de exhibiciones, espectáculos y espacios de formación. Estos ámbitos tienen como eje nodal la búsqueda de nuevas modalidades de trabajo y de vínculos dentro de las instituciones, estableciendo agendas de intereses y prioridades alternativas a las que impone el mercado, y apostando por un trabajo colectivo.

Q. Libet afirma la necesidad de un involucramiento topológico con el espacio al momento de la ocupación, al estilo de un estratega, "preguntándose: ¿Cuáles son sus agujeros, entradas, salidas? ¿Cómo puede uno desalienarlo, desidentificarlo, hacerlo inoperante, hacerlo común?" [Steyerl, 2009: 120]. Por otra parte, gran parte de la historiografía local [Giunta, 2009; Labaké, 2010; Longoni, 2010] coincide en marcar el año 2001 como el brote de una serie de iniciativas artísticas autogestionadas, de unas emergencias de sensibilidad colectiva que pretenden borrar la lógica establecida por el mercado a través del trabajo colaborativo y la modalidad de organización horizontal, así como el establecimiento de una agenda artística alternativa a la propuesta por las grandes instituciones.

\footnotetext{
3 Ejemplo de esto son las tomas de la Universidad de Buenos Aires, la UNA, o el CONICET.

4 Información disponible en: http://

www.teatrovalleoccupato.it/chi-siamo

5 Información disponible en: http:/ / www.

nuovocinemapalazzo.it/chi-siamo/

6 Información disponible en: http://latabacalera.net/

7 Información disponible en: http:/ / www.zku-berlin.org/
} 


\section{Una casa}

El uso de la palabra casa en el título del proyecto puede pensarse más allá de la referencia al nombre de la institución -Casa nacional del Bicentenario- y es posible remitirlo a la idea de un hogar, de espacio cotidiano, donde los artistas parecieran vivir, criar $^{8}$, y trabajar en conjunto entre ellos, los demás trabajadores de la institución y los espectadores. La convivencia es una condición sine qua non, lo cual dificulta la diferenciación entre la obra de un artista y otro. Los espacios fueron ocupados por creadores que muchas veces superponen sus obras o modifican lo que otro hizo previamente, por lo tanto no hay un planteo de obras como unidades independientes, sino como un gran trabajo en comunidad. Al estilo de un palimpsesto, se generan distintas capas que acumulan trabajos de diversa autoría: "el que viene tiene que vérselas con los rastros del anterior, tal como en una casa ocupada donde te las arreglas con lo que encontrás"9 . La referencia al hogar también está presente en "la construcción concreta de ambientes momentáneos de vida" [Debord, 1957: 1] a lo largo y a lo ancho de los tres pisos que forman este proyecto. Vislumbramos una cocina, un taller de costura, dormitorios públicos, distintos livings o lugares de estar, un jardín de invierno, una biblioteca popular y una peluquería, entre otros. La idea del lugar de pertenencia, del hogar, de un espacio identitario propio también reflexiona y denuncia los problemas habitacionales, la desprotección que sufre la gran cantidad de personas que no cuentan con un hogar ni con el apoyo estatal para lograr dicho objetivo. A su vez, el espacio físico como representación de la identidad también se vincula con la obra presentada por Florencia Levy acerca de la ocupación del territorio palestino y su desplazamiento cultural.

El proyecto cuenta, además, con un espacio destinado a una "oficina de publicaciones" abierta, libre, en la que cada individuo, sea empleado, artista, o espectador -si vale referirse a estas categorías aún- puede acercarse y tomar de manera gratuita cualquier ejemplar para llevárselo o leerlo en el living creado alrededor del escritorio de trabajo. Suerte de marco teórico ${ }^{10}$, este conjunto de textos

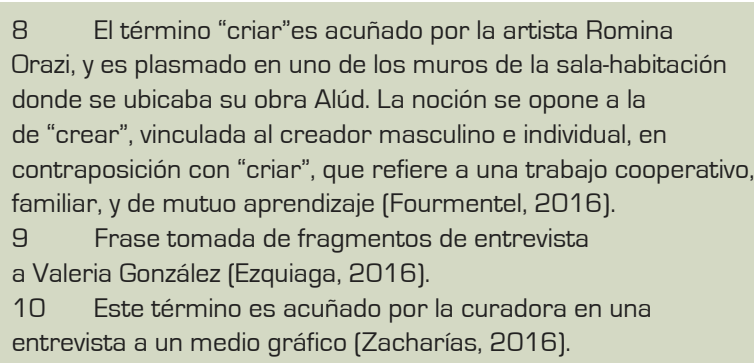

publicados y distribuidos refieren a diversas problemáticas que Casa Tomada pretende poner en evidencia. Entre los dieciséis números, encontramos textos y fragmentos variopintos de autores como Michel de Certeau, Allan Kaprow, George Didi Huberman y Jacques Rancière.

Casa Tomada mantuvo un aspecto de taller constante, de nunca mostrarse preparada para la contemplación plena, sino erigirse como un organismo en preparación o mutación permanente. Se utiliza una estética de precariedad deliberada, que evidencia el estado de denuncia, comenzando con los ventanales de la fachada blanqueados a la cal [Fotografía 1]. No hubo vernissage, inauguración para la prensa, ni cierre de la muestra. De hecho, no hay infografías que den cuenta del nombre de cada obra o artista, el anonimato se impone. El proyecto también apela al humor y juego, el gesto irreverente y la ironía se hacen presentes en diversas formas. Un lateral de la fachada presenta un cartel de grandes dimensiones que anuncia que el New York Times calificó a Casa Tomada como la muestra del año. En otro rincón de la exhibición, hojas de papel impresas penden de hilos transparentes; esos papeles llevan inscritos los comentarios realizados en la web del tradicional diario "La Nación" por indignados lectores, irritados por el dudoso uso -a su entender- que Casa Tomada hace del espacio público, refiriéndose en particular, a través de frases agresivas y súmamente peyorativas a la obra, Alúd de Romina Orazi [Fotografía 5], quien simula un enorme montículo de tierra en plena sala. Estas hojas se ubicaron estratégicamente en un pequeño espacio que aloja los andamios, las pruebas del artificio de la obra de Orazi, que demuestran que ese montículo de tierra que parece hundir sus profundas redes en el edificio no es más que una puesta en escena muy efectiva.

La CNB también se alza contra los postulados clásicos de los espacios de exhibición y de las instituciones jerarquizadas en las que un organigrama dicta el rol a cada uno de los integrantes de la misma. Gran parte de los empleados del espacio fue obligado a asumir un nuevo rol y apropiarse a su manera del proyecto. Valeria González señala que "los guardias de sala no cuidan, sino que alientan a participar, sacan fotos y comparten los mates. El departamento de producción es ahora una oficina de publicaciones que reparte fotocopias de lecturas para pensar" [2016a]. Otras áreas como prensa o producción quedaron desprovistas de sus tareas habituales, por lo que debieron concebir otras. Un grupo de trabajadores creó una oficina abierta en la sala dedicada a mostrar una suerte de archivo de la institución, donde exponían los diversos papeles guardados, acumulados en el 
establecimiento y los exhibían tratando de buscar un orden, un patrón, un sentido que pueda vincularlos o justificar su existencia dentro de la institución. Las conclusiones de este trabajo mutaban a medida que pasaban los días. Siguiendo esta concepción del trabajo, uno de los ascensores del recinto presenta desprolijamente escrita la frase "Todas las inteligencias son iguales" [Fotografía 4). Este aspecto resulta notable ya que trasciende la instancia de arte participativo, el cual se tornó moneda corriente en el terreno del arte. La desjerarquización de los roles, la incitación a la búsqueda de los propios intereses, de nuevas funciones y vínculos permite vislumbrar renovadas modalidades de lo institucional. Como señala González, "Al irse los empleados volvieron las personas, y tuvieron que hacerse preguntas dignas de artistas, diría Deleuze" [Zacharías, 2016]. Uno de los textos distribuidos por la Oficina de Publicaciones de Casa Tomada expone la necesidad de modelar nuevas formas de hacer en las instituciones sociales, enlazando la práctica crítica con la operatividad material, a través del caso de los servicios de salud en Trieste. Franco Salvini [2016] introduce el concepto de umbral como espacio liminar entre la institución y su entorno social, donde es posible construir dispositivos de composición, "donde puede empezar la invasión, el lugar donde la transición puede convertirse en irreversible" [2016: २).

\section{El proceso, la ocupación, la situación}

Retomando la idea de la muestra como un proceso permanente, el texto ¿curatorial? introductorio al proyecto señala la importancia de la noción del trabajo vivo, "siempre en gerundio: es en tanto se está realizando" [Anónimo, 2016]. Casa Tomada también pretende poner en debate cómo se define el trabajo del artista en la sociedad, cómo se diferencia de otras labores, cómo es retribuido ese tiempo como fuerza de trabajo y transformado en mercancía, “¿Cuándo, en qué momento exactamente comienza a haber arte? ¿Qué umbrales atraviesa una actividad entre el taller y el museo? ¿Cuánta energía se pierde en el camino? ¿Qué bisturí separa un objeto de arte de los restos del hacer?" [Anónimo, 2016]. ${ }^{11}$ Estas reflexiones acerca del estatuto social del trabajo del artista

11 Las referencias al texto curatorial -si cabe utilizar dicha nominación- presentado en la web de la CNB son citadas con autor anónimo debido a la elección de la institución de no asignarle la autoría de dicho texto a ningún integrante del proyecto en particular. ya habían sido ensayadas por la curadora "clandestina"12 y directora de la CNB, Valeria González en el proyecto Ostinato en 2006. Junto a un grupo de artistas, González propuso utilizar el espacio de exposición -en ese caso Estudio Abierto, espacio de gestión estatal al igual que CNB- pensando "cómo tornar los obstáculos en medios, el poder que se ejerce sobre nosotros en potencia productiva" [González, 2006: 2].

Hito Steyerl aborda esta cuestión proponiendo una distinción entre las nociones de trabajo y ocupación, afirmando que el primero es "una labor, implica un comienzo, un producto, y eventualmente un resultado. El trabajo es visto primordialmente como el medio para un fin: un producto, una recompensa, o un salario. Es una relación instrumental. También produce a un sujeto por medio de la alienación”, mientras que una ocupación:

[...] es lo contrario. Una ocupación mantiene a las personas ocupadas en vez de darles una labor pagada. Una ocupación no está articulada a un resultado; no tiene una conclusión necesaria. Como tal, no conoce de la alienación tradicional, ni de alguna correspondiente idea de subjetividad. Una ocupación no necesariamente supone una remuneración, ya que se entiende que el proceso contiene su propia gratificación. No tiene un marco temporal excepto el mismo paso del tiempo. No se centra en un productor/trabajador, pero incluye a consumidores, reproductores [...] cualquiera que busque distracción o involucramiento [Steyerl, 2014: 108].

Casa Tomada parece estar sosteniendo el proceso permanente como esencia de su labor, desde la noción de ocupación de Steyerl, no como un medio para llegar a un fin, a una obra terminada, digna de ser contemplada, sino como un fin en sí mismo, "lo que solía materializarse exclusivamente como objeto o producto, como trabajo de arte, ahora tiende a aparecer como actividad o performance" [Steyerl, 2014: 110]. De esta manera, la labor del artista pasa a ser una ocupación, al igual que su obra.

A su vez, Reinaldo Ladagga, en su texto "Una Asamblea" [2006], incluido dentro de las publicaciones distribuidas por la Casa, analiza la formulación del film "La Comunidad" de Peter Watkins. El autor señala la concepción peculiar del trabajo del director, quien desarrolló un exhaustivo trabajo de investigación sobre el proceso de filmación y de vinculación de los distintos actuantes, primando la labor colectiva, la improvisación, y sometiendo el set a

12 Este término es acuñado por la curadora en una entrevista a un medio gráfico [Zacharías, 2016] 
largos lapsos de tiempo de filmación para lograr una experiencia más integrada. En palabras de Watkins "este método de filmación dinámico y experiencial los forzaba a abandonar la pose y el artificio, y los conducía a un cuestionamiento de la sociedad contemporánea, que tenían que confrontar en el sitio mismo" [2006: 168]. El texto plantea la relevancia de la instancia del proceso, en la que que los límites entre forma y proceso se funden, la forma hace posible que el proceso ocurra, pero sin el proceso la forma carece de sentido [2006: 166]. Resulta interesante entonces concebir los múltiples proyectos y gestos de Casa Tomada como parte de un proceso que crea formas borrosas y nunca totalmente terminadas, aunque altamente poderosas y polisémicas. A su vez, la noción de proceso puede pensarse también en términos de Debord como situación, como una acción continuada, donde los acontecimientos serán efímeros y se construirán como meros "lugares de pasaje", equivalentes a las ruinas del espectáculo moderno, expulsando al espectador de su rol pasivo y llevándolo a la actividad, provocando sus capacidades para revolucionar su propia vida [Debord, 1957: 4].

Desde disímiles marcos teóricos se pretende poner el acento en la acción. Del mismo modo, una inscripción en las paredes de la Casa anunciaba "pensar la política del campo del arte como lugar de trabajo. Se trata de mirar lo que el arte hace en vez de lo que muestra".

\section{Consideraciones finales}

El posicionamiento político del hacer, la crítica institucional y las prácticas colaborativas conforman en Casa Tomada un núcleo indivisible. La toma, lindante entre la legalidad y la ilegalidad, opera como un recurso sorpresivo, atractivo, de fácil reconocimiento y de sencilla aprehensión para cualquier habitante de la Ciudad de Buenos Aires. La toma es el grito de denuncia sobre el conflicto, una herramienta para alzar la voz y poner en evidencia una situación. Como instancia de protesta, debe ser acompañada por una etapa sucesiva -no necesariamente posterior en sentido temporal- de apropiación, construcción y reconfiguración, con la recuperación de ese espacio desde otra perspectiva, ocupar "también significa desmilitarizarlo -por lo menos en términos de jerarquía - para luego militarizarlo de manera distinta" [Steyerl, 2014: 120]. Retomando, entonces, las preguntas iniciales, cabe señalar que quizá el valor del proyecto no radique en su avance antiinstitucional, sino en la mostración de mecanismos alternativos de vinculación y de trabajo, en la evidencia de una concepción renovada de dichas instancias, del brote de una serie de prácticas sensibles aplicadas a otros terrenos sociales, capaces de ser apropiada a los dominios del arte bajo sus propios preceptos, todavía en construcción. Casa Tomada no busca destruir la institución sino rescatarla, reconfigurarla a tiempo para que no se convierta en un mausoleo ${ }^{13}$. La revolución vanguardista ya no es el objetivo, esa tarea inconclusa de la Modernidad no tiene vigencia en la contemporaneidad, sino más bien se aspira a "pensar cómo las diferentes y ambivalentes prácticas de los que viven dentro y a través de la institución pueden contribuir a fortalecer una práctica social de emancipación" [Salvini, 2016: 6]. Quizá la vía de la reconfiguración de las instituciones sea la certera para arribar en el arte del autogobierno, en la invención de formas duraderas de organización social democrática [Hardt y Negri, 2011). Ahora resta preguntarse qué sucederá en el futuro con la Casa después de un proceso tan significativo.

\section{Referencias}

Anónimo [2016]. Casa Tomada, texto curatorial. Publicado únicamente en la web: http:/ / www.casadelbicentenario. gob.ar/33905/agenda-y-noticias/noticias/exposicioncasa-tomada. Buenos Aires.

AAV [2010]. Poéticas contemporáneas. Itinerarios en las artes visuales en la Argentina de los 90 al 2010. Buenos Aires: Fondo Nacional de las Artes.

Carman, María y María Paula Yacovino [2007].

"Transgrediendo el derecho de los que nos vulneran: Espacios ocupados y recuperados en la Ciudad de Buenos Aires". En Rev. argent. sociol. [online] vol.5, n.8 [consultado 2017-01-18], pp. 28-50. Disponible en: <http:/ / www. scielo.org.ar /scielo.php?script=sci_arttext\&pid=S1669$32482007000100003 \&$ lng $=e s \& n r m=i s o>$.

Debord Guy [1997]. "Rapport sur la construction des situations et sur les conditions de l'organisation de l'action de la tendance situationnista internationale", Conferencia fundacional de la Internacional Situacionista de Coscio d'Arroscia, julio/1957. En Internationale Situationniste. París: Librairie Artheme Fayard.

Ezquiaga, Mercedes [2016]. "Del objeto de arte al hacer, la Casa Nacional del Bicentenario fue apropiada por artistas", Telam Cultura, Publicada el 27/9/2016. Disponible en: http:/ / www.telam.com.ar/notas/201609/165044-delobjeto-de-arte-al-hacer-la-casa-nacional-del-bicentenario-fue-

13 Este término es acuñado por la curadora en una entrevista a un medio gráfico [Zacharías, 2016]. 
apropiada-por-artistas.html

Fourmentel, Ignacio [2016]. "Un Alúd irrumpe en el museo: Romina Orazi en Casa Tomada". UNTREF piensa Casa Tomada. Disponible en: https:/ / cnbcasatomada.files. wordpress.com/2016/12/ignacio-fourmentel.pdf

Giunta, Andrea [2009]. Poscrisis. Arte Argentino después del 2001. Buenos Aires: Siglo Veintiuno Editores.

González, Valeria [2006]. Ostinato. Texto inédito. Buenos Aires.

Hardt, Michael y Negri, Antonio [2014]. "El devenir príncipe de la multitud”, Commonwealth, Madrid: Akal. Traducción Raúl Sánchez Cedillo.

Labaké, Andrés [2010]. "Algunas ciudades y otras historias. Fragmentos y transcripciones. Espacios y grupos de artistas autogestionados", en AAV, Poéticas contemporáneas. Itinerarios en las artes visuales en la Argentina de los 90 al 2010. Buenos Aires: Fondo Nacional de las Artes.

Ladagga, Reinaldo [2006]. "Una Asamblea”, en Estética de la Emergencia, Buenos Aires: Adriana Hidalgo.

Longoni, Ana [2010]. "Tres coyunturas del activismo artístico en la última década”, en AAW, Poéticas contemporáneas. Itinerarios en las artes visuales en la Argentina de los 90 al 2010. Buenos Aires: Fondo Nacional de las Artes.

Salvini, Franco [2016]. Instituir el umbral, Viena. Disponible en: trasversal.at/transversal/0916/editorial/impressum. Traducción: Kike España Naveira.

Steyerl, Hito [2014]. "El arte como ocupación: declaraciones para una autonomía de la vida", en Los condenados de la pantalla, Buenos Aires: Caja Negra Editora.

Zacharías, María Paula [2016]. "Artistas okupas: La casa del Bicentenario los invita a sentirse como en casa", Diario La Nación, Publicada el 27/9/16. Disponible en: http:/ / www.lanacion.com.ar/1941594-artistas-okupas-la-casadel-bicentenario-los-invita-a-sentirse-como-en-casa 